\title{
DEFICIÊNCIA AUDITIVA INFANTIL: IMPLICAÇÕES E SOLUÇÕES
}

\author{
Infantile hearing loss: implications and solutions
}

\author{
Cladi Inês Gatto ${ }^{(1)}$, Tania Maria Tochetto ${ }^{(2)}$
}

\section{RESUMO}

Objetivo: promover uma abordagem teórica dos aspectos relevantes a respeito da audição na infância e da detecção precoce da deficiência auditiva infantil. Métodos: foi realizada pesquisa bibliográfica sobre a importância da audição no desenvolvimento infantil, o histórico da detecção precoce da deficiência auditiva infantil e a metodologia usual nos programas de triagem auditiva neonatal. O período de análise foi a partir de 1980 e os descritores utilizados foram deafness, hearing loss, hearing impairment and children. Resultados: a audição é o elemento fundamental para a aquisição e desenvolvimento da linguagem. Muitos são os indicadores de risco que podem afetar a audição nos períodos pré e peri natal. Sendo a detecção precoce um fator determinante para o prognóstico de reabilitação, é de extrema importância a sua efetivação. Existem diferentes metodologias para a detecção da deficiência auditiva, porém os programas de triagem auditiva neonatal que utilizam emissões otoacústicas vêm demonstrando grande aceitação pela sua eficácia e praticidade. Conclusão: existem graves implicações da deficiência auditiva para o desenvolvimento infantil. A implementação dos programas de triagem auditiva neonatal pode garantir a detecção precoce, o diagnóstico e a reabilitação a tempo de minimizar os efeitos da deficiência auditiva sobre o indivíduo.

DESCRITORES: Audição; Perda Auditiva; Triagem Neonatal

\section{INTRODUÇÃO}

A linguagem desempenha um papel essencial na organização perceptual, na recepção e estruturação das informações, na aprendizagem e nas interações sociais do ser humano.

A audição constitui-se em um pré-requisito para a aquisição e o desenvolvimento da linguagem. Audição e linguagem são funções correlacionadas e interdependentes.

Um dos principais distúrbios que podem interferir no desenvolvimento da linguagem e da fala é a deficiência auditiva. A American Speech-Language-Hearing Association ${ }^{1}$ considera que a deficiência auditiva representa $60 \%$ dos distúrbios da comunicação.

Da população mundial com idade inferior a 15 anos, aproximadamente 62 milhões têm perda audi-

(1) Fonoaudióloga da Clínica São Braz de Passo Fundo; Mestre em Distúrbios da Comunicação Humana pela Universidade Federal de Santa Maria.

(2) Fonoaudióloga; Professora do Departamento de Fonoaudiologia da Universidade Federal de Santa Maria; Doutora em Ciências dos Distúrbios da Comunicação Humana pela Universidade Federal de São Paulo. tiva permanente ${ }^{2}$; dois terços (41 milhões) habitam países em desenvolvimento ${ }^{3}$. Em neonatos a incidência de deficiência auditiva é de 1,5 a 5,95 por 1000 nascimentos ${ }^{4}$.

Estudo de base populacional, inédito no Brasil e na América Latina, em Canoas (RS), município com aproximadamente 200 mil habitantes, constatou perdas auditivas leves em 19,3\% dos sujeitos entrevistados e perdas auditivas incapacitantes em $6,8 \%{ }^{5}$.

A perda auditiva é a deficiência congênita mais freqüente e mais prevalente dentre aquelas rotineiramente triadas em programas de saúde preventivos ${ }^{6}$.

Os períodos críticos de maturação das funções biológicas do ser humano acontecem nos primeiros meses de vida. Assim, a identificação e intervenção precoces da surdez possibilitam ao deficiente auditivo alcançar desempenho comunicativo muito próximo ao das crianças ouvintes ${ }^{7-9}$. Estudos nacionais e internacionais apontam que a deficiência auditiva tem sido diagnosticada tardiamente, quando prejuízos irreversíveis ao desenvolvimento da criança já ocorreram ${ }^{10}$.

Há muito tempo metodologias para identificar precocemente a deficiência auditiva infantil têm sido estudadas. Com a descoberta das Emissões Otoacústicas (EOA) ${ }^{11}$ e o desenvolvimento de equi- 
pamentos de manipulação simples e prática, vêm se vislumbrando a inserção do profissional da Fonoaudiologia nas equipes de neonatologia dos hospitais e maternidades com o intuito de implementar os programas de Triagem Auditiva Neonatal (TAN). Além de executar a TAN é preciso disponibilizar procedimentos de diagnóstico para as crianças que faIharem neste procedimento inicial e reabilitação para aquelas diagnosticadas como surdas.

Apesar da reconhecida importância da triagem auditiva na detecção e intervenção precoce dos casos de deficiência auditiva, muitos esforços ainda precisam ser empreendidos a fim de que ela se torne uma realidade nacional e universal.

No Brasil a TAN é regulamentada por lei em 13 municípios: Americana, Campinas, Itapeva, Jahu e São Paulo (SP); Brasília (DF); Campo Grande (MS); Florianópolis (SC); Juiz de Fora e Uberlândia (MG); Porto Alegre e Santa Maria (RS); Rio de Janeiro (RJ). Os estados brasileiros que dispõem de lei determinando a obrigatoriedade da TAN são Paraná, Pernambuco e São Paulo. O projeto de lei 03.842, tramitando desde 1997, prevê a realização do exame de EOA, gratuitamente, em todos os hospitais e maternidades públicas e privadas do País ${ }^{12}$.

O objetivo deste estudo foi promover uma abordagem teórica dos aspectos relevantes a respeito da audição na infância e da detecção precoce da deficiência auditiva infantil.

\section{MÉTODOS}

Foi realizada pesquisa bibliográfica em artigos de periódicos nacionais e internacionais, livros e documentos eletrônicos considerando-se a relevância e o valor informativo de tais materiais para o assunto em estudo.

Foram consultados livros científicos da área de Fonoaudiologia, sites oficiais de entidades vinculadas à audiologia e periódicos das áreas de Fonoaudiologia, Otorrinolaringologia e Pediatria da base de dados MEDLINE, por meio dos descritores deafness, hearing loss, hearing impairment and children, publicados a partir de 1980.

O tema foi apresentado considerando-se os seguintes tópicos: a importância da audição no desenvolvimento infantil, o histórico dos procedimentos de detecção precoce da deficiência auditiva infantil, a relevância das emissões otoacústicas como metodologia usual nos programas de triagem auditiva neonatal e os indicadores de risco que podem afetar a audição no período neonatal.

\section{RESULTADOS}

A detecção precoce da deficiência auditiva Os primeiros relatos do uso de estímulos sono- ros para a obtenção de respostas auditivas em bebês datam de 1944 com a descrição de respostas comportamentais como piscadas de olhos e flexões da cabeça em direção ao som, as quais se tornariam mais definidas com a maturação biológica ${ }^{13}$.

A aplicabilidade de diversos procedimentos de respostas comportamentais e automáticas foi testada em recém-nascidos. Em 1956, Wedenberg realizou um estudo sobre o reflexo cócleo palpebral (RCP), afirmando que a presença do mesmo poderia ser um indicador da audição normal em neonatos ${ }^{14}$.

O primeiro procedimento automático ficou conhecido como "crib-o-gram". No berço da criança eram colocados transdutores capazes de detectar movimentos associados à apresentação de estímulos sonoros $^{13}$.

Ainda em 1974 foi utilizada a avaliação de Potencial Evocado Auditivo do Tronco Encefálico (PEATE) para testar a audição de crianças entre três meses e três anos de idade. Devido à confiabilidade e a estabilidade das respostas, o procedimento possibilitaria avaliar a audição em crianças de forma objetiva ${ }^{13}$.

Em 1978, um importante fato marcou as descobertas no campo da Audiologia. Kemp detectou uma "liberação de energia sonora produzida na cóclea que se propagava pela orelha média até o meato acústico externo". Denominou-a Emissão Otoacústica (EOA) ${ }^{11}$. A presença de EOA representaria o funcionamento adequado das células ciliadas externas da cóclea. Essa descoberta, associada ao desenvolvimento de equipamentos de manipulação simples e prática, deu início a uma nova fase na avaliação auditiva de neonatos. Por meio desse procedimento, a triagem auditiva pode ser implementada de acordo com seu sentido mais amplo, ou seja, um processo de se aplicar a um grande número de indivíduos medidas rápidas e simples, que irão identificar aqueles com alta probabilidade de portar um distúrbio na função testada ${ }^{13}$.

Johnsen, Bagi e Elberling, em 1983, foram os primeiros a investigar a possibilidade de utilização do teste de EOA como meio de triagem auditiva em recém-nascidos, acreditando ser um procedimento rápido, não evasivo, de fácil aplicabilidade, com alta sensibilidade e especificidade para detectar alterações no sistema auditivo periférico ${ }^{15}$.

A confiabilidade das EOA foi demonstrada por Bonfils, Uziel e Pujol em 1988. Os autores compararam os limiares de resposta para a onda $V$ no Potencial Evocado Auditivo do Tronco Encefálico com a presença de EOA em bebês. Comprovaram que as EOA sempre estiveram presentes quando o limiar da onda $\mathrm{V}$ foi igual ou inferior a $30 \mathrm{~dB}$. Ao contrário, quando o limiar da onda $V$ foi superior a $40 \mathrm{~dB}$, as EOA estavam ausentes. Eles apontaram as EOA como um novo caminho para a investigação da audição periférica em bebês e crianças, salientando que a técnica é simples, rápida, não invasiva e objetiva ${ }^{16}$. 
A descoberta das EOA ocasionou o surgimento de uma nova visão sobre a função da cóclea, pois mostrou que ela é capaz não apenas de receber sons, mas também de produzir energia acústica ${ }^{17}$. Por meio das EOA, seria possível estudar os aspectos mecânicos da função coclear de forma não invasiva e objetiva, independente do potencial da ação neural. Dessa maneira, informações objetivas poderiam ser obtidas sobre os elementos pré-neurais da cóclea ${ }^{17}$.

A primeira experiência clínica de ampla escala com triagem auditiva por meio de EOA foi descrita por White, Vohr e Behrens em 1993. Neste programa de triagem auditiva neonatal universal realizado em Rhode Island, nos Estados Unidos, foram avaliados 1850 recém-nascidos de berçário comum e 304 de UTI Neonatal ${ }^{4}$.

A partir dos resultados mencionados por diversos autores o Joint Committee on Infant Hearing (1994) ${ }^{18}$ passou a recomendar a triagem auditiva neonatal universal por métodos fisiológicos (PEATE e/ou EOA), no momento da alta hospitalar, a fim de permitir a identificação da perda auditiva antes dos três meses de idade e a intervenção clínico-educacional até os seis meses de idade. Em 2000, uma nova reunião desse comitê estabeleceu os princípios e referenciais para o controle da efetividade dos programas de Triagem Auditiva Neonatal Universal ${ }^{19}$.

Atualmente, os programas de triagem auditiva neonatal estão amplamente difundidos e aplicados com características específicas em cada país. Os resultados já podem ser identificados por meio da redução da idade média de detecção da deficiência auditiva em muitos locais ${ }^{20}$. Apesar desses avanços, a triagem auditiva neonatal ainda não representa uma realidade universal e totalmente efetiva, principalmente nos países em desenvolvimento ${ }^{3}$.

No Brasil os primeiros programas de Triagem Auditiva iniciaram em 1987, na Universidade Federal de São Paulo, então Escola Paulista de Medicina (SP) e na Universidade Federal de Santa Maria (RS). Ambos os programas utilizavam procedimentos comportamentais. Em 1988, no Hospital Israelita Albert Einstein (SP), foi implantado programa de Triagem Auditiva com Potenciais Evocados de Tronco Encefálico (PEATE) ${ }^{12}$.

Os primeiros esforços pela divulgação da TAN datam de 1998, com a criação do Grupo de Apoio à Triagem Auditiva Neonatal Universal (GATANU) ${ }^{6}$ e do Comitê Brasileiro sobre Perdas Auditivas na Infância (CBPAI) ${ }^{21}$. Em 1999 o CBPAl emitiu a primeira recomendação brasileira sobre identificação, manejo, reabilitação e habilitação das perdas auditivas na infância ${ }^{21}$.

No ano seguinte a Sociedade Brasileira de Pediatria (SBP) criou uma Força Tarefa para a prevenção da deficiência auditiva ${ }^{22}$.

A falta de esclarecimento e conscientização da população em geral assim como dos profissionais da área da saúde que atuam diretamente com os neonatos representam entraves para a detecção precoce da deficiência auditiva infantil em nosso país.

Considera-se importante ressaltar a diferença existente entre os procedimentos de triagem auditiva e de diagnóstico audiológico. A triagem auditiva emprega testes de varredura para detectar um possível problema auditivo. O principal objetivo da triagem auditiva é identificar os possíveis casos de perda auditiva. Já na etapa de diagnóstico são utilizados vários testes para determinar os níveis de audição da criança, o tipo e a provável etiologia da perda auditiva, o prognóstico e o tratamento adequado.

\section{Emissões Otoacústicas (EOA)}

As emissões otoacústicas são sons gerados dentro da cóclea normal, tanto espontaneamente (Emissão Otoacústica Espontânea) ou como resposta a uma estimulação acústica (Emissão Otoacústica Evocada) ${ }^{24}$.

As emissões otoacústicas são sons mensurados no canal auditivo externo, originários da energia mecânica produzida pela expansibilidade e contratilidade das células ciliadas externas ${ }^{25}$. Tais respostas podem ocorrem na ausência de estimulação externa (Emissões Otoacústicas Espontâneas) durante ou depois de estimulação acústica (Emissões Otoacústicas Evocadas) ${ }^{24}$. Tal energia é transmitida de forma reversa através da orelha média e da membrana timpânica e pode ser captada no conduto auditivo externo.

Apesar de não quantificar a deficiência auditiva, a ausência de EOA permite o topodiagnóstico da lesão: o órgão de Corti ${ }^{11}$.

As aplicações clínicas das emissões otoacústicas podem ser ${ }^{26}$. triagem auditiva para a detecção precoce da perda auditiva em recém-nascidos; - avaliação objetiva da perda neurossensorial de origem coclear versus retrococlear; - monitoramento audiológico de pacientes expostos a agentes agressores como drogas ototóxicas e ruídos intensos; - perda súbita com flutuação da audição; - exame objetivo que pode ser aplicado a pacientes pouco colaboradores.

A importância clínica das EOA advém do fato de a maioria das patologias causadoras de surdez neurossensorial, nomeadamente as associadas à surdez infantil, apresentarem, como lesão primária ou secundária, disfunção das células ciliadas externas ${ }^{27}$. É bem conhecida a susceptibilidade deste receptor celular aos efeitos adversos de doenças virais e bacterianas, alterações genéticas, agentes externos, tais como sons intensos, drogas ototóxicas e químicas ${ }^{28}$.

As emissões otoacústicas evocadas subdividemse de acordo com o estímulo utilizado para evocálas. São elas: Emissões Otoacústicas Evocadas Tran- 
sitórias, Emissões Otoacústicas Produto de Distorção e Emissões Otoacústicas Freqüência Dependente.

As Emissões Otoacústicas Evocadas Transitórias (EOAET) resultam da estimulação acústica de toda a cóclea por click, estímulo acústico breve que abrange ampla gama de freqüências. A presença de resposta sugere limiares auditivos inferiores a $30 \mathrm{~dB}^{27}$. AS EOAET são registradas em $98 \%$ dos indivíduos com audição normal ${ }^{17}$.

A técnica mais empregada e recomendada na Triagem Auditiva Neonatal tem sido a de EOAET por utilizar estímulos acústicos de fraca intensidade, abranger uma vasta gama de freqüências e por finalizar o registro, num curto período de tempo, geralmente em torno de um minuto por orelha ${ }^{29}$.

A pesquisa de EOA vem se firmando como método de eleição nos programas de Triagem Auditiva Neonatal por apresentarem maior sensibilidade e especificidade na detecção das alterações da audição, se comparada aos métodos comportamentais. Além disso, o teste de EOA possibilita detectar perdas auditivas leves e unilaterais, o que não ocorre com o uso de métodos comportamentais.

Comparada ao registro de PEATE, as EOA representam um método de custo inferior, mais prático e mais rápido.

É importante salientar que a captação das EOAE sofre influência de alterações da orelha média, de fatores ambientais, como o ruído excessivo e de ruídos oriundos do organismo do bebê ${ }^{24,30}$.

Resultados falso-positivos podem causar ansiedade nos pais até que o diagnóstico efetivo seja estabelecido. Essa ansiedade, porém, foi considerada pequena e não apresentou repercussões negativas na relação mãe-bebê ${ }^{31}$. Os efeitos negativos dos resultados alterados poderiam ser minimizados por meio de esclarecimentos sobre o teste ${ }^{32}$. Os efeitos negativos oriundos da Triagem Auditiva Neonatal podem ser considerados aceitáveis, em vista dos benefícios da detecção precoce da deficiência auditiva sobre o desenvolvimento do indivíduo ${ }^{33}$.

Indicadores de risco associados à deficiência auditiva

As intercorrências pré, peri ou pós-natais podem aumentar a probabilidade de desvios no desenvolvimento global infantil e podem afetar, inclusive, a audição. Os dados da OMS revelam que $40 \%$ das gestantes têm alguma complicação. No Brasil, 36,5\% dos casos de deficiência auditiva em crianças foram associados a intercorrências durante a gestação ${ }^{34}$.

A ocorrência de deficiência auditiva bilateral significativa foi encontrada em um a três em cada 1000 nascidos vivos saudáveis e dois a quatro em cada 100 recém-nascidos provenientes das unidades de tratamento intensivo ${ }^{4}$.

A atenção dispensada à avaliação auditiva de crianças portadoras de indicadores de risco para a per- da auditiva é bastante apropriada, porém, sabe-se que cerca de 30 a $50 \%$ das crianças com deficiência auditiva não poderiam ser enquadradas nos critérios de risco ${ }^{35}$. Destaca-se assim a importância de a triagem auditiva ser universal.

As ocorrências passíveis de apresentarem efeitos deletérios sobre a audição vêm sendo investigadas há longa data. Já em 1982, o Joint Committee on Infant Hearing ${ }^{36}$ preconizou o registro de alto risco, por meio do qual, as crianças que apresentassem algum dos critérios estabelecidos deveriam ser avaliadas antes dos três meses de idade e nunca após os seis meses. Em 1994, o Comitê revisou o documento anterior, publicado em 1982, modificando a denominação de "critérios de risco" para "indicadores de risco" ${ }^{18}$.

Mais recentemente, o Joint Committee on Infant Hearing ${ }^{19}$ estabeleceu os seguintes indicadores de risco para deficiência auditiva para neonatos até 28 dias: a) Doença ou condição que exija internação em UTI por 48 horas ou mais; b) Sinais ou outros achados associados a síndromes que conhecidamente incluam a deficiência auditiva neurossensorial ou condutiva; c) História familiar de deficiência auditiva permanente; d) Anomalias crânio-faciais, incluindo aquelas com anormalidades da orelha; e) Infecções congênitas como citomegalovírus, herpes, toxoplasmose ou rubéola.

Sabendo que determinadas intercorrências podem implicar em deficiência auditiva, as gestantes devem ser orientadas sobre meios de prevenir problemas auditivos.

\section{DISCUSSÃO}

Por tratar-se de uma privação sensorial de graves conseqüências para a criança, a família e a sociedade, e ainda por apresentar elevada incidência e prevalência ${ }^{21}$, a deficiência auditiva tem sido objeto de preocupações e estudos na busca de alternativas que possam minimizar seus efeitos deletérios sobre o desenvolvimento social, emocional e cognitivo do indivíduo.

A deficiência auditiva é invisível até que seus efeitos traduzam-se no comprometimento da linguagem oral. Quando o período de otimização das estruturas neurológicas para essa função já foi concluído, a reabilitação é menos eficaz. Desta forma, a detecção precoce dessa deficiência é o aspecto fundamental na determinação do prognóstico terapêutico ${ }^{7}$.

Os procedimentos para a detecção precoce da deficiência auditiva têm evoluído significativamente. Atualmente as emissões otoacústicas representam um método de grande aceitação mundial nos programas de Triagem Auditiva Neonatal ${ }^{29}$.

Apesar dos avanços tecnológicos possibilitarem a detecção da deficiência auditiva a partir das primeiras 24 
horas de vida, ainda se constata importante atraso na suspeita e no diagnóstico da surdez ${ }^{16}$. A pouca valorização da prevenção primária e secundária, a falta de conscientização e informação da população sobre a necessidade e importância da detecção precoce da surdez infantil e a reduzida participação das equipes de saúde, principalmente dos pediatras na valorização e no encaminhamento para Triagem Auditiva Neonatal são possivelmente os fatores responsáveis pelo quadro que aí está.

Programas de triagem auditiva, além das etapas obrigatórias de diagnóstico e intervenção poderiam também envolver informação e conscientização dos profissionais e da população em geral.

Padronizar os protocolos de TAN e criar base de da- dos (nacional, estadual, municipal) ${ }^{21}$, seria o passo inicial para suprir a escassez de dados sobre a perda auditiva no País. Se viável, a notificação compulsória da deficiência auditiva poderia subsidiar a formulação e avaliação das políticas, planos e programas de saúde auditiva ${ }^{12}$.

\section{CONCLUSÃO}

A literatura pesquisada aponta graves implicações da deficiência auditiva para o desenvolvimento infantil. A implementação dos programas de triagem auditiva neonatal pode garantir a detecção precoce, o diagnóstico e a reabilitação a tempo de minimizar os efeitos da deficiência auditiva sobre o indivíduo.

\begin{abstract}
Purpose: to promote a theoretical approach of the relevant aspects regarding the hearing in childhood and the early detection of the infantile hearing loss. Methods: bibliographical research on the importance of hearing in infantile development, the description of the early detection of the hearing loss and usual methodology in the programs of newborn hearing screening was carried through. The period of analysis went from 1980 and the key-words were deafness, hearing loss, hearing impairment and children. Results: the hearing is the basic element for the acquisition and development of language. Many are the risk indicators that can affect hearing in the pre and peri native periods. Being the early detection a determinative factor for the rehabilitation prognostic, its effectuation is of extreme importance. There are different methodologies for detecting hearing loss, however the programs of newborn hearing screening through otoacoustic emissions have been demonstrating effectiveness and feasibility. Conclusion: there are serious implications of hearing loss for infantile development. The implementation of the programs of newborn hearing screening can ensure early detection, diagnosis and rehabilitation in timely-manner to minimize the effect of hearing loss on the subject.
\end{abstract}

KEYWORDS: Hearing; Hearing Loss; Neonatal Screening

\section{REFERÊNCIAS}

1. American Speech-Language-Hearing Association. Committee on Infant Hearing: guidelines for audiologic screening of newborn infants who are at risk for hearing impairment. ASHA. 1989; 31:89-92.

2. Smith A. Preventing deafness: an achievable challenge. The $\mathrm{WHO}$ perspective. International Congress Series 2003; 1240:183-91.

3. Olusanya BO, Luxon LM, Wirz SL. Detection of permanent childhood hearing loss in a developing country. 3 February 2005. Disponível em: URL:http:// www.saferhealthcare.org.uk/ihi.

4. White RK, Vohr BR, Behrens TR. Universal newborn hearing screening using transient evoked otoacoustic emissions: results of the rhode island hearing assessment project. Semin Hear. 1993; 14:18-29.

5. Béria JU, Raymann BCW, Gigante LP, Figueiredo AL, Jota G, Roithmann R, et al. Perda auditiva incapacitante e fatores sócio-econômicos: um estu- do de base populacional em Canoas, RS, Brasil. In: 19- Encontro Internacional de Audiologia; 2004 29/ 04-02/05; Bauru, São Paulo.

6. Grupo de Apoio à Triagem Auditiva Neonatal Universal - GATANU. Disponível em URL: http:// www.gatanu.org.

7. Yoshinaga-Itano C, Sedey AL, Coulter DK, Mehl A. Language of early and later identified children with hearing loss. Pediatrics. 1998; 102(5):1161-71.

8. Garcia CFD, Isaac ML, Oliveira JAA. Emissão otoacústica evocada transitória: instrumento para detecção precoce de alterações auditivas em recémnascidos a termo e pré-termo. Rev Bras Otorrinolaringol. 2002; 68(3):344-52.

9. Colunga JCM, Méndez JCA, Villarreal JMC, Zapico MJA, Estrada CM, Alvarez MLF. Despistage de la hipoacusia neonatal: resultados después de 3 años de iniciar nuestro programa. Acta Otorrinolaringol. Esp. 2005; 55:55-8.

10. Erenberg A, Lemons J, Sia C, Trunkel D, Ziring P. 
Newborn and infant hearing loss: detection and intervention: task force on newborn and infant hearing. Pediatrics. 1999; 103(2):527-30.

11. Kemp DT. Stimulated acoustic emissions from within the human auditory system. J Acoust Soc Am. 1978; 64(5):1386-91.

12. Tochetto T, Vieira EP. Legislação brasileira sobre triagem auditiva neonatal. Carapicuíba: Pró-fono; 2006. $58 \mathrm{p}$.

13. Northern JL, Downs MP. A audição em crianças. São Paulo: Manole; 1989. 421 p.

14. Wedenberg E. Auditory tests on newborn infants. Acta Otoryngol. 1956; 46(5):446-61.

15. Johnsen NJ, Bagi P, Elberling C. Evoked acoustic emissions from the human ear. III: findings in neonates. Scand Audiol. 1983; 12(1):17-24.

16. Bonfils P, Uziel A, Pujol R. Screening for auditory dysfunction in infants by evoked oto-acoustic emissions. Arch Otolaryngol Head Neck Surg. 1988; 114(8):887-90.

17. Probst R. Otoacoustic emissions: an overview. Adv Otorhinolaryngol. 1990; 44:1-91.

18. Joint Committee on Infant Hearing. Joint Committee on Infant Hearing 1994 Position Statement. Pediatrics. 1995; 95(1):152-6.

19. Joint Committee on Infant Hearing. Year 2000 position statement: principles and guidelines for early hearing detection and intervention programs. Am J Audiol. 2000; 9(1):9-29.

20. Lotke M. Hearing Impairment . Disponível em: URL: http://www.emedicine.com/ped/topic931.htm.

21. Comitê Brasileiro sobre Perdas Auditivas na Infância (CBPAI). Recomendações 01/99 do Comitê Brasileiro sobre Perdas Auditivas na Infância. J Cons Fed Fonoaudiol. 2000; 5:3-7.

22. Segre CAM. I Encontro Nacional de Triagem Auditiva Neonatal; 2003 5-7 de setembro de 2003; Hospital Sírio-Libanês, São Paulo. Disponível em: URL: h t t p : / / w w w. gat a n u . org / a tualidades / PALESTRASIENCONTRO/forcatarefaconceicao.pdf 23. McCormick, B. Screening for hearing impairment in young children. London: Croom Helm; 1988. 120 p. 24. Norton SJ, Stover LJ. Emissões otoacústicas: um novo instrumento clínico. In: Katz J. Tratado de audiologia clínica. São Paulo: Manole; 1999. p. 444-58.
25. Vono-Coube CZ, Costa Filho OA. Emissões otoacústicas: uma visão geral. In: Frota S. Fundamentos em fonoaudiologia. Rio de Janeiro: Guanabara-Koogan; 1998. p. 95-106.

26. Parrado MES. Emissões otoacústicas em recémnascidos. In: Bassetto MCA, Brock $R$, Wajnsztejn R. Neonatologia: um convite à atuação fonoaudiológica. São Paulo: Lovise; 1998. p. 295-300. 27. Oliveira P, Castro F, Ribeiro A. Surdez infantil. Rev Bras Otorrinolaringol. 2002; 68(3):417-23.

28. Guedes MC, Passos SN, Gomes MVSG, Bento RF. Estudo da reprodutibilidade das emissões otoacústicas em indivíduos normais. Rev Bras Otorrinolaringol. 2002; 68(1):34-8.

29. Bassetto MCA, Chiari BM, Azevedo MF. Emissões otoacústicas transientes (EOAET): amplitude da resposta em recém-nascidos a termo e pré-termo. Rev Bras Otorrinolaringol. 2003; 69(1):84-91.

30. Cerruti VQ. Estudo das emissões otoacústicas evocadas em neonatos- transients e produto de distorção [doutorado]. São Paulo (SP): Universidade de São Paulo; 2001.

31. Hergils L, Hergils A. Universal neonatal hearing screening: parental attitudes and concern. Br J Audiol. 2000; 34(6):321-7.

32. Weichbold V, Welzl-Muller K. Universal neonatal hearing screening: attitude and fears of mothers. HNO. 2000; 48(8):606-12.

33. Grandori F, Lutman M. The European consensus development conference on neonatal hearing screening (Milan, May 15-16, 1998). Am J Audiol. 1999; 8(1):19-20.

34. Nóbrega M. Aspectos diagnósticos e etiológicos da deficiência auditiva em crianças e adolescentes [mestrado]. São Paulo (SP): Escola Paulista de Medicina; 1993.

35. National Institutes of Health. Early identification of Hearing Impairment in Infants and Young Children - Consensus Development Conference Statement. March 1-3, 1993. Disponível em: URL: http:// c ons e n s us.n i h.gov/ $1993 /$ 1993HearingInfantsChildren092html.htm.

36. American Academy of Pediatrics. Joint Committee on Infant Hearing - Position Statement 1982. Pediatrics. 1982; 70(3):496-7.

RECEBIDO EM: 14/08/06

ACEITO EM: 04/12/06

Endereço para Correspondência:

Rua Diogo de Oliveira, 103

Passo Fundo - RS

CEP: $99025-130$

Tel: (54) 33141308

E-mail: cladi.gatto@terra.com.br 